\title{
A Practically Simple and Easy Approach for Minimizing the Influence of Fresnel Fringes on Phase Sensitivity Measured from Electron Holography
}

\author{
Zhouguang Wang
}

Micron Technology Inc., Boise, Idaho, USA

Electron holography has been applied for practical semiconductor device analysis for years. To precisely map p-n junction distributions, especially those with low doping levels in the devices, a high phase sensitivity measured from reconstructed phase image is required. Besides the fringe contrast and number of electrons in recorded hologram, an important factor that affects the phase sensitivity is Fresnel fringes. Fig. 1(a) is a typical hologram taken with no sample and the profile on the right shows the intensity modification by Fresnel fringes. This can be seen more clearly in the reconstructed amplitude image (Fig. 1(b)). The influence of Fresnel fringes on the phase is shown in Fig. 1(c). The phase profile indicates that a phase fluctuation up to $0.26 \mathrm{rad}$ is induced by Fresnel fringes. Generally, two kinds of approaches can be utilized to reduce the effects of the fluctuation on the phase sensitivity: one is experimentally obtaining a hologram without Fresnel fringes [1, 2] and the other is numerically correcting [3]. These methods, however, need either special equipments or lengthy and complicated calculation, hard to be adapted for the use in semiconductor industry where a quick turn-around time is required. Practically we have employed a much simple and easy method to minimize the influence of Fresnel fringes.

As shown in Fig. 1, the modification of Fresnel fringes on the intensity of interference fringes attenuates from the edge to the center of the interference band. If only the center part is used, the influence of the Fresnel fringes will be minimized. Figs. 2(a), (b), (c) and (d) are a series of reference holograms taken by a GIF CCD camera at biprism voltages of $15 \mathrm{~V}, 25 \mathrm{~V}, 35 \mathrm{~V}$ and $45 \mathrm{~V}$ respectively. The corresponding reconstructed amplitude images are given in Figs. 2(e), (f), (g) and (h) to show the influence of the Fresnel fringes. It can be seen clearly that with increasing biprism voltage the interference band expands, more and more Fresnel fringes move out of the recorded hologram. When only center part is recorded as shown in Figs. 2(c) and (d), the influence of the Fresnel fringes almost disappears completely. A one-dimensional Si/Si p-n junction with very low doping level was used for the evaluation of the method. Two holograms, A and B including their reference holograms were collected using a $2 \mathrm{~K} \times 2 \mathrm{~K}$ GIF camera. Hologram A was imaged using the edge part of the interference band, in which some Fresnel fringes were included. Hologram B, on the other hand, was formed by using only the center part of the interference band. Both holograms are reconstructed by using same parameters, and the resulting phase images and their corresponding profiles drawn along depth direction are shown respectively in Figs. 3(a) and (b). Although a reference hologram has been used for the reconstruction, the phase fluctuation induced by Fresnel fringes can be seen obviously in Fig. 3(a) so that no p-n junction can be recognized. In Fig. 3(b), however, there is no influence of the Fresnel fringes, as the result, we can clearly see a phase shift between $\mathrm{p}$ - and n-doped regions even the shit is very small $(<0.1 \mathrm{rad})$. The phase measurements in $\mathrm{Si}$ area yield the standard deviations of $0.162 \mathrm{rad}$ and 
$0.065 \mathrm{rad}$, respectively for Figs. 3(a) and (b), which explains why the p-n junction can be mapped successfully in the latter case.

\section{References:}

[1] K. Yamamoto, T. Hirayama and T. Tanji, Ultramicroscopy 10 (2004), p265.

[2] K. Harada, A. Tonomura, Y. Togawa, T. Akashi and T. Matsda, Appl. Phys. Lett. 84(2004), p3229.

[3] K. Yamamoto, I. Kawajiri, T. Tanji, M. Hibino and T. Hirayama, J. Electr. Microsc. 49(2000), p31.

Figure 1. A typical hologram with Fresnel fringes (a) and its reconstructed amplitude (b) and phase (c) images. Their profiles drawn from red box clearly show the influence of the Fresnel fringes on both amplitude and phase.
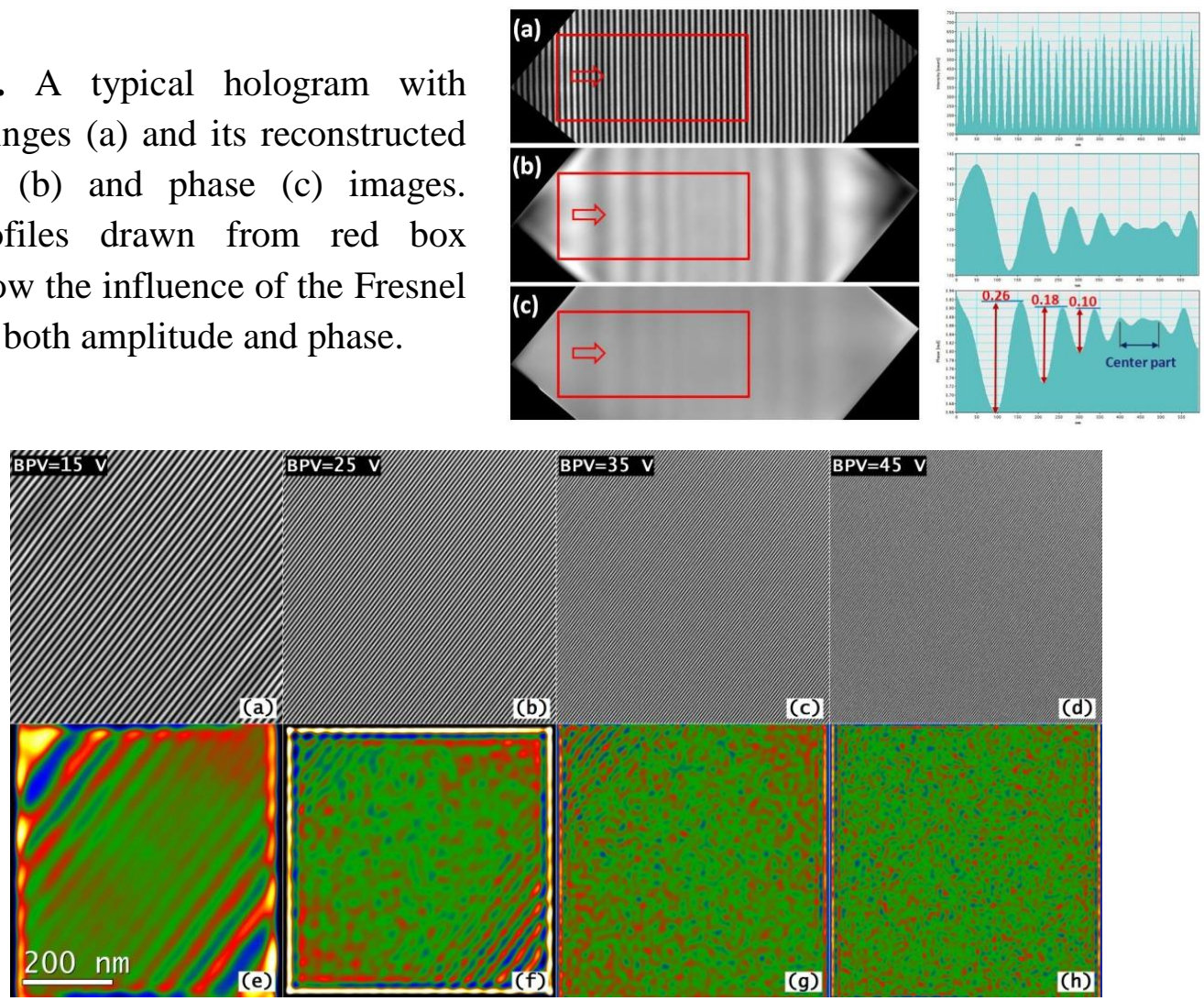

Figure 2. A series of holograms taken at different biprism voltages and their corresponding reconstructed amplitude images showing the reduction of the influence of Fresnel fringes.
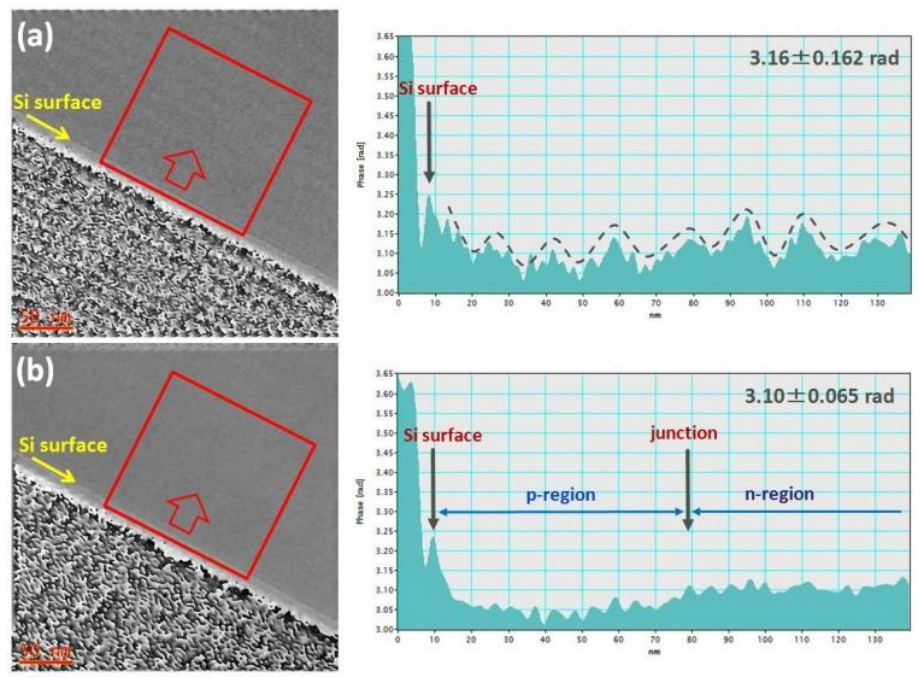

Figure 3. Electron holography for a 1D $\mathrm{Si} / \mathrm{Si}$ p-n junction with very low doping level. (a) the phase image reconstructed from a common hologram with Fresnel fringes, the profile from it showing the influence of the Fresnel fringes on the phase; (b) the phase image from a hologram using only center part of the interference band, no Fresnel fringe effect so its profile revealing $\mathrm{p}-\mathrm{n}$ junction location. 